\title{
CN Zeeman measurements in star formation regions
}

\author{
E. Falgarone ${ }^{1}$, T. H. Troland ${ }^{2}$, R. M. Crutcher ${ }^{3}$, and G. Paubert ${ }^{4}$ \\ 1 LERMA/LRA, CNRS UMR 8112, École Normale Supérieure and Observatoire de Paris, 24 rue Lhomond, \\ 75231 Paris Cedex 05, France \\ e-mail: edith@lra.ens.fr \\ 2 University of Kentucky, Department of Physics and Astronomy, Lexington, KY 40506, USA \\ e-mail: troland@pa.uky.edu \\ 3 University of Illinois, Department of Astronomy, Urbana, IL 61801, USA \\ e-mail: crutcher@uiuc.edu \\ 4 IRAM, 7 avenida Divina Pastora, Granada, Spain \\ e-mail: paubert@iram.es
}

Received 14 February 2008 / Accepted 17 May 2008

ABSTRACT

\begin{abstract}
Aims. Magnetic fields play a primordial role in the star formation process. The Zeeman effect on the $\mathrm{CN}$ radical lines is one of the few methods of measuring magnetic fields in the dense gas of star formation regions.

Methods. We report new observations of the Zeeman effect on seven hyperfine CN $N=1-0$ lines in the direction of 14 regions of star formation.

Results. We have improved the sensitivity of previous detections, and obtained five new detections. Good upper limits are also achieved. The probability distribution of the line-of-sight field intensity, including non-detections, provides a median value of the total field $B_{\text {tot }}=0.56 \mathrm{mG}$ while the average density of the medium sampled is $n\left(\mathrm{H}_{2}\right)=4.5 \times 10^{5} \mathrm{~cm}^{-3}$. We show that the CN line probably samples regions similar to those traced by CS and that the magnetic field observed mostly pervades the dense cores. The dense cores are found to be critical to slightly supercritical with a mean mass-to-flux ratio $M / \Phi \sim 1$ to 4 with respect to critical. Their turbulent and magnetic energies are in approximate equipartition.
\end{abstract}

Key words. magnetic fields - stars: formation - ISM: molecules - turbulence - polarization - ISM: kinematics and dynamics

\section{Introduction}

The role of magnetic fields in the formation of structure in dense molecular clouds and in the star formation process remains unclear (see Crutcher 2007, for a recent review). If sufficiently strong, magnetic fields may support clouds against gravitational collapse and thus prevent or delay star formation. Shu et al. (1999), Mouschovias \& Ciolek (1999), and MacLow \& Klessen (2004) have reviewed the theory. Magnetic fields appear to provide the only viable mechanism for transporting angular momentum from collapsing cores, and they may play a significant role in the physics of bipolar outflows and jets that accompany protostar formation (Cabrit 2007). Observation of magnetic fields in molecular clouds is therefore crucial.

Crutcher (2007) reviewed the various techniques and results for studying magnetic fields in molecular clouds. Of these techniques, the Zeeman effect provides the only direct method for measuring magnetic field strengths in molecular clouds. To date, detections of the Zeeman effect in the interstellar medium have been made only in lines of $\mathrm{H} \mathrm{I}, \mathrm{OH}, \mathrm{CN}$, and $\mathrm{H}_{2} \mathrm{O}$. Thermal lines of the first two species probe relatively low-density gas $n(\mathrm{H})<10^{4} \mathrm{~cm}^{-3}$. $\mathrm{OH}$ and $\mathrm{H}_{2} \mathrm{O}$ maser emission lines probe high densities, but in special regions - very localized in space and perhaps shock compressed. As a tracer of high density gas, the $\mathrm{CN}$ thermal lines probe dense regions in molecular clouds and $\mathrm{CN}$ Zeeman observations are therefore a unique tool for measuring magnetic field strengths in star formation regions.
The radio transitions of $\mathrm{CN}$ have been discussed extensively by Turner \& Gammon (1975). The Einstein $A$ of the strongest CN hyperfine component within the $\lambda=3 \mathrm{~mm} N=1 \rightarrow 0$ transition is $A=1.19 \times 10^{-5} \mathrm{~s}^{-1}$, which means that the $N=1 \rightarrow 0$ transition of $\mathrm{CN}$ offers the opportunity to measure magnetic fields in the density range $10^{4}$ to $10^{6} \mathrm{~cm}^{-3}$, the density of molecular cores that may be in transition from equilibrium (between gravity and magnetic/turbulent support) to collapse to form stars.

Previously, a successful series of CN Zeeman observations was carried out with the IRAM 30-m telescope, with a quarterwave plate polarimeter (Crutcher et al. 1996, 1999). In this paper we report additional results obtained with the IRAM-30 m telescope with a new correlation spectropolarimeter. We both improved the sensitivity of some of the previous results and obtained data on new sources.

\section{The CN Zeeman effect}

Table 1 lists the 7 strongest $\mathrm{CN}$ hyperfine lines together with each line's frequency, relative intensity, Zeeman splitting coefficient, and relative sensitivity to the Zeeman effect (the product of the Zeeman splitting coefficient and the relative intensity). The fact that the $N=1 \rightarrow 0$ transition of $\mathrm{CN}$ has 7 strong hyperfine components (there are actually 9 components, but 2 are much weaker than the others and hence are not useful for Zeeman observations) with very different Zeeman splitting factors is essential for the success of $\mathrm{CN}$ Zeeman measurements. 
Table 1. CN $N=1-0$ hyperfine lines.

\begin{tabular}{ccccc}
\hline \hline Line & $v(\mathrm{GHz})$ & $\mathrm{RI}^{a}$ & $Z(\mathrm{~Hz} / \mu \mathrm{G})^{b}$ & $\mathrm{RI} \times Z^{c}$ \\
\hline 1 & 113.144 & 8 & 2.18 & 17.4 \\
2 & 113.171 & 8 & -0.31 & 2.5 \\
3 & 113.191 & 10 & 0.62 & 6.2 \\
4 & 113.488 & 10 & 2.18 & 21.8 \\
5 & 113.491 & 27 & 0.56 & 15.1 \\
6 & 113.500 & 8 & 0.62 & 5.0 \\
7 & 113.509 & 8 & 1.62 & 13.0 \\
\hline
\end{tabular}

${ }^{a} \mathrm{RI}$ is the relative intensity of each hyperfine component.

${ }^{b} Z$ is the Zeeman splitting factor of each hyperfine component.

${ }^{c} \mathrm{RI} \times Z$ is the relative sensitivity to $B_{\mathrm{los}}$.

Instrumental polarization such as beam squint (a two-lobe pattern in the circularly polarized primary telescope beam), polarized sidelobes, et cetera will typically produce a Stokes $V$ signal in the $\mathrm{CN}$ spectra that is comparable to or larger than the Zeeman signal. There is no way to avoid this for extended emission, and it would be extremely difficult, if not impossible, to eliminate these instrumental polarization artifacts. The main effect of instrumental polarization is to produce a pseudo-splitting of the spectral line that appears to mimic Zeeman splitting. However, instrumental polarization does not know about the Zeeman effect. The CN Zeeman splitting factor (Crutcher et al. 1996) varies quite significantly among the 7 hyperfine components (see Table 1). It is possible to observe all 7 hyperfine lines simultaneously and to fit the 7 observed Stokes $V$ spectra to the expression: $V_{i}(v)=C_{1} I_{i}(v)+C_{2} \frac{\mathrm{d} I_{i}(v)}{\mathrm{d} v}+C_{3} Z_{i} \frac{\mathrm{d} I_{i}(v)}{\mathrm{d} v}$, where $i=1$ to 7 for the 7 hyperfine components and $Z_{i}$ is the Zeeman splitting factor for each hyperfine line. $C_{1}$ absorbs any gain difference between left and right polarization and any linearly polarized line signal. $C_{2}$ absorbs any instrumental polarization effects that produce pseudoZeeman splitting. $C_{3}$ is non-zero only if there is circular polarization line splitting due to the CN Zeeman effect. Crutcher et al. (1996) tested this fitting procedure with simulated noisy data and found that it is robust; for example, a $2 \sigma$ Zeeman signal can be reliably extracted from data with more than an order of magnitude larger instrumental polarization artifacts. For CN Zeeman observations the polarimeter need not be perfect (as indeed the quarter-wave plates used previously were definitely not), and polarized sidelobes that would severely affect attempts to measure polarization of extended emission or other instrumental polarization effects do not prevent success.

\section{Observations}

Observations were carried out with the IRAM-3 0m telescope, which has a beam width of 23" at this frequency, in May 2004, 2005, 2006 and 2007. In order to observe at the peak CN line positions, we first made very short Stokes $I$ maps (generally 5-point maps). For our observations the two orthogonally polarized heterodyne receivers were made coherent by sharing reference synthesizers. The signals from the receivers were fed to the Vespa correlator which performed the auto-correlations and cross-correlations of the signals from both receivers. This gave 4 spectra - the power spectra of the horizontally and vertically (in the receiver cabin) polarized receivers, and the real and imaginary parts of the cross-correlations (i.e., the horizontally polarized receiver correlated with the vertically polarized receiver).

The spectra were first converted to temperature scale by applying the standard calibration procedure for spectral observations. The next step was to correct for the phase errors: while the receivers are coherent, they exhibit an unknown phase difference (due to slightly different optical paths, unmatched cable lengths, absolute phase of the spectrometers) which must be taken into account. This difference was measured by taking an additional spectrum on a cold load through a polarization grid during the calibration procedure. The uncertainty on the phase correction in each individual channel was about $0.1^{\circ} \mathrm{rms}$. It was also very stable with time, varying by less than $1^{\circ}$ per hour.

The phase correction was applied to the cross-correlation spectra to obtain 4 spectra in the receiver cabin domain (Horizontal, Vertical, Real and Imaginary). Stokes $I$ is Horizontal+Vertical and the imaginary part of the crosscorrelation spectra is Stokes $V$, the circular polarization component. We therefore obtained Stokes $I$ and $V$ spectra for each source. Although Stokes $U$ and $Q$ spectra were potentially available, we did not make use of these data.

\section{Results}

Together with the earlier $\mathrm{CN}$ Zeeman observations, there are now sensitive $\mathrm{CN}$ Zeeman observations toward 14 positions. CN Zeeman results for OMC1n4 are from Crutcher et al. (1996) and for OMC1s, DR21OH1, and DR21OH2 are from Crutcher et al. (1999). Results for other positions are based on the observations reported here, both of new sources and additional integrations on previously observed positions to improve the sensitivity.

Table 2 gives positions and various physical parameters for the CN Zeeman sources. Appendix A gives additional notes on the individual clouds, including especially the references for the density and radius of each. $T_{\mathrm{A}}^{*}$ is the observed line strength of the strongest hyperfine component, line 5 in Table $1 . v$ is the line peak velocity with respect to the local standard of rest, and $\Delta v$ is the full line width at half maximum intensity. Distances $(d)$ are taken from the literature. The radius of the $\mathrm{CN}$ sources we observe $(r)$ are determined from the distances and the measured angular sizes of $\mathrm{CN}$ emission if available, or other tracers such as CS if not. We assumed that $\mathrm{CN}$ and the other high density tracers are co-located, although only higher angular resolution (interferometer) mapping can test this assumption.

Simon (1998) mapped many of the CN Zeeman clouds with the IRAM-30 m telescope in both the $\mathrm{CN} N=1 \rightarrow 0$ and $N=2 \rightarrow 1$ lines; we determine radii from his maps whenever possible. The volume densities of $\mathrm{H}_{2}$ in the regions where $\mathrm{CN}$ is observed are taken from the literature, usually from analysis of the excitation of CS lines; the critical density of the $J=2 \rightarrow 1 \mathrm{CS}$ transition is $n \approx 8 \times 10^{4} \mathrm{~cm}^{-3}$, very similar to that of the $N=1 \rightarrow 0 \mathrm{CN}$ transition (Turner \& Gammon 1975) (see Appendix A for details).

The column density of $\mathrm{H}_{2}$ has been estimated in two ways. (1) $N_{n, r}\left(\mathrm{H}_{2}\right)$ comes from $n\left(\mathrm{H}_{2}\right)$ and the core radius, assuming a spherical core. (2) $N_{\mathrm{CN}}\left(\mathrm{H}_{2}\right)$ comes from our inference of the column density of $\mathrm{CN}$ molecules, as follows. Our spectra show that although the measured relative intensities of the 7 hyperfine components vary slightly from their optically thin LTE ratios (Table 1), the variations are not large, suggesting that the $\mathrm{CN}$ lines are optically thin. We checked this quantitatively as follows. We assumed LTE, which implies that, except for line optical depth effects, the relative strengths of the observed lines should be those given in Table 1 . Lines 3 and 4 have the same relative intensity, so we averaged these observed line strengths and obtained the observed strength of relative intensity 10 lines from this average. We then computed the ratio of the observed strengths of line 5 to the line 3 and 4 average. For a very large 
Table 2. CN Zeeman sources - positions and physical parameters.

\begin{tabular}{|c|c|c|c|c|c|c|c|c|c|c|c|c|}
\hline Cloud & $\alpha(2000)$ & $\overline{\delta(2000)}$ & $\begin{array}{l}T_{\mathrm{A}}^{*} \\
\mathrm{~K}\end{array}$ & $\begin{array}{c}v \\
\mathrm{~km} \mathrm{~s}^{-1}\end{array}$ & $\begin{array}{c}\Delta v \\
\mathrm{~km} \mathrm{~s}^{-1}\end{array}$ & $\begin{array}{c}d \\
\mathrm{kpc}\end{array}$ & $\begin{array}{c}r \\
\mathrm{pc}\end{array}$ & $\begin{array}{c}n\left(\mathrm{H}_{2}\right) \\
10^{5} \mathrm{~cm}^{-3}\end{array}$ & $\begin{array}{c}N_{n, r}\left(\mathrm{H}_{2}\right) \\
10^{23} \mathrm{~cm}^{-2}\end{array}$ & $\begin{array}{c}N_{\mathrm{CN}}\left(\mathrm{H}_{2}\right) \\
10^{23} \mathrm{~cm}^{-2}\end{array}$ & $\begin{array}{c}M_{\mathrm{obs}} \\
M_{\odot}\end{array}$ & $\begin{array}{c}M_{\text {vir }} \\
M_{\odot}\end{array}$ \\
\hline W3OH & 022704.1 & 615222 & 2.2 & -46.5 & 4.2 & 2.4 & 0.12 & 1.5 & 1.1 & 0.9 & 88 & 430 \\
\hline OMC1s & 053513.5 & -052252 & 14.7 & 8.5 & 2.0 & 0.45 & 0.03 & 18 & 3.4 & 2.3 & 17 & 26 \\
\hline OMC1n1 & 053514.5 & -052207 & 13.0 & 9.9 & 1.4 & 0.45 & 0.03 & 18 & 3.6 & 1.5 & 16 & 13 \\
\hline OMC1n4 & 053516.8 & -051931 & 14.5 & 9.4 & 1.5 & 0.45 & 0.03 & 14 & 2.8 & 2.2 & 18 & 15 \\
\hline NGC2024 & 054144.2 & -015541 & 8.6 & 11.0 & 2.2 & 0.45 & 0.04 & 5.9 & 1.4 & 2.2 & 18 & 40 \\
\hline S255 & 061253.7 & 175922 & 5.6 & 7.3 & 2.3 & 2.5 & 0.18 & 3.7 & 4.2 & 1.3 & 500 & 200 \\
\hline G10.6 & 181028.7 & -195549 & 8.9 & 2.8 & 5.8 & 6.5 & 0.19 & 14 & 16.4 & 4.9 & 2100 & 1300 \\
\hline M17SWHI & 182022.9 & -161132 & 17.3 & 19.9 & 4.3 & 1.3 & 0.11 & 6.0 & 4.2 & 6.2 & 430 & 440 \\
\hline M17SWCN & 182025.0 & -161342 & 16.9 & 19.6 & 3.3 & 1.3 & 0.10 & 6.0 & 3.7 & 5.3 & 290 & 230 \\
\hline $\mathrm{S} 106 \mathrm{OH}$ & 202728.4 & 372241 & 5.3 & -1.7 & 2.0 & 1.7 & 0.07 & 1.0 & 0.4 & 0.9 & 17 & 55 \\
\hline S106CN & 202729.5 & 372254 & 7.3 & -1.8 & 1.9 & 1.7 & 0.07 & 1.0 & 0.4 & 1.3 & 21 & 50 \\
\hline DR21OH1 & 203859.9 & 422238 & 4.5 & -4.7 & 2.3 & 3 & 0.15 & 1.7 & 1.5 & 1.0 & 170 & 160 \\
\hline DR21OH2 & 203859.9 & 422238 & 2.6 & -0.9 & 2.3 & 3 & 0.15 & 1.7 & 1.5 & 0.7 & 140 & 160 \\
\hline S140 & 221917.1 & 631835 & 6.0 & -6.3 & 2.4 & 0.9 & 0.05 & 6.0 & 1.0 & 1.3 & 28 & 63 \\
\hline
\end{tabular}

line optical depth, this ratio should be 1 ; for a very small line optical depth, the ratio will be 2.7. All but one of these ratios are within the range 2.7-1 expected for LTE line strengths and zero to infinite line optical depth; the one that is not has the $\mathrm{RI}=10$ lines only very slightly too weak for LTE and low optical depth. The maximum line optical depth found by this technique is $\tau \approx 0.5$. We therefore compute the column density in the $N=0$ state assuming the RI $=10$ lines are optically thin (see Turner \& Gammon 1975). We then compute the total column density of $\mathrm{CN}$ in all states by assuming that all states are excited with an assumed excitation temperature of $25 \mathrm{~K}$. (These are warm, dense cores, and several of the $T_{\mathrm{A}}^{*}$ are not too far below $25 \mathrm{~K}$ in strength.) We then assume $\mathrm{CN} / \mathrm{H}_{2}=4 \times 10^{-9}$ in order to find $N_{\mathrm{CN}}\left(\mathrm{H}_{2}\right)$. This value of $\mathrm{CN} / \mathrm{H}_{2}$ is consistent with those found by Turner \& Gammon (1975) in dense, warm cores, and matches the results found in OMC1 cores by Johnstone et al. (2003). Finally, we compute the observed masses $M_{\mathrm{obs}}$ of the $\mathrm{CN}$ Zeeman sources from the radii and geometric mean of $N_{n, r}\left(\mathrm{H}_{2}\right)$ and $N_{\mathrm{CN}}\left(\mathrm{H}_{2}\right)$, denoted $N_{23}\left(\mathrm{H}_{2}\right)$ in the following. We also list for comparison the virial masses $M_{\mathrm{vir}}=210 r \Delta v^{2} M_{\odot}$, where $r$ is expressed in pc and $\Delta v$ in $\mathrm{km} \mathrm{s}^{-1}$.

As an example of the data, Fig. 1 shows the spectra of $\mathrm{W} 3 \mathrm{OH}$. The Stokes $I$ spectrum is the average (weighted by the sensitivity to the Zeeman effect) of hyperfine lines 1, 4, 5, and 7 (Table 1); these are the lines that have significant sensitivity to the Zeeman effect. The Stokes $V$ spectrum is the equivalent average, where the non-Zeeman contributions to the observed $V$ due to gain imbalance and instrumental polarization (coefficients $C_{1}$ and $C_{2}$ in the fitting equation (Sect. 2)) have been removed. For $\mathrm{W} 3 \mathrm{OH}$ the instrumental polarization contribution to Stokes $V$ is the equivalent of a $5.6 \mathrm{mG}$ Zeeman signal for a (totally artificial) $Z=1 \mathrm{~Hz} / \mu \mathrm{G}$ for all 7 hyperfine components. Hence, the instrumental polarization contribution in this case is about 5 times greater than the true Zeeman signal. Only the large variation in the Zeeman splitting factors among the hyperfine components makes it possible to obtain reliable $B_{\text {los }}$ results from CN Zeeman observations, as discussed in Sect. 2. Overplotted on Stokes $V$ is $\mathrm{d} I / \mathrm{d} v$ computed from the average Stokes $I$ spectrum and scaled to the fitted magnetic field strength, $B_{\mathrm{los}}=+1.10 \mathrm{mG}$.

In Table 3, we list the line-of-sight magnetic field strength $B_{\text {los }}$ and the $1 \sigma$ uncertainty in each measurement. Instrumental polarization effects have been eliminated from the Stokes $V$ spectra by the fitting procedure, so the uncertainty in each measurement is dominated by stochastic noise. Earlier during the series of CN Zeeman observations (Crutcher et al. 1996)

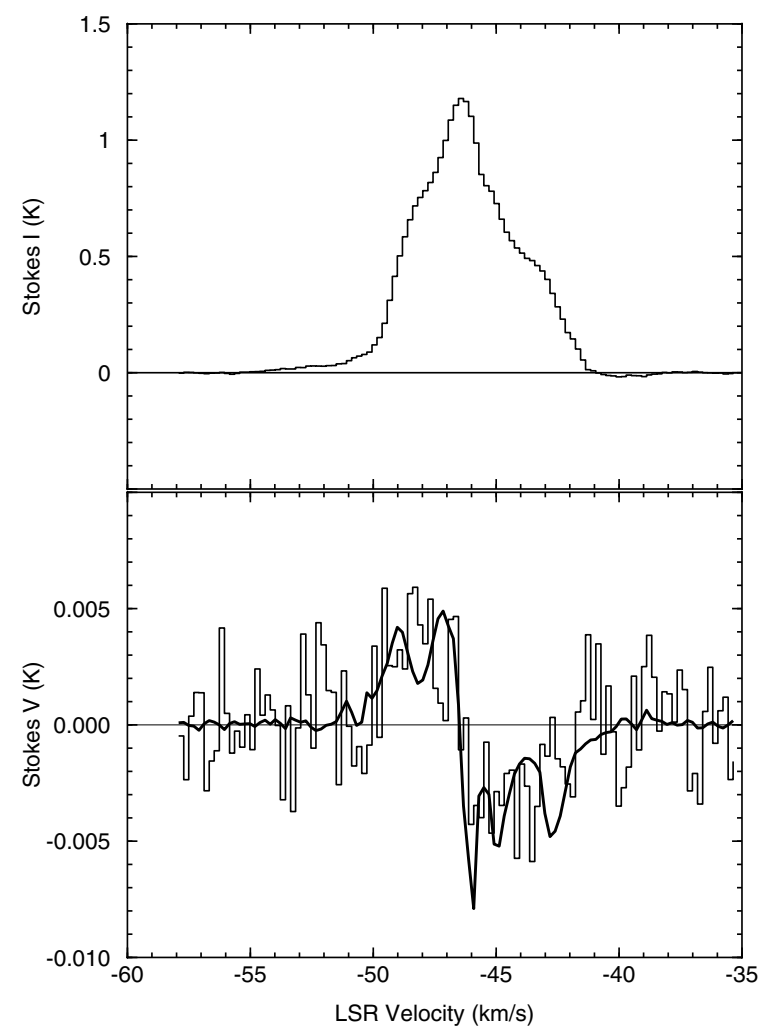

Fig. 1. W3OH CN Zeeman spectra. The top plot is the Stokes parameter $I$ spectrum, and the bottom plot is the Stokes parameter $V$ spectrum (histogram) and $\mathrm{d} I / \mathrm{d} v$ (heavy line) scaled for $B_{\mathrm{los}}=+1.10 \mathrm{mG}$.

we tested the Zeeman fitting procedure by simulating the fitting process with artificially generated spectral lines with various $B_{\text {los }}$ and random spectral noise. We then fitted the resulting spectra to test what signal-to-noise ratio was required to achieve a reliable detection of $B_{\text {los }}$. We found that the results followed the normal probability distribution function, so at the $2 \sigma$ level $4.6 \%$ of the measurements would be false positives. For 14 measurements (the number of cloud measurements reported here), one would then expect 0.6 false "detections" of $B_{\text {los. }}$. We therefore adopted $2 \sigma$ as the statistically valid cut off for claiming detections. Our lowest signal-to-noise ratio is slightly above $2 \sigma$. Therefore, the detections we claim here are all probably real, although it is possible (although statistically unlikely) that 1 or even 2 of the 
Table 3. CN Zeeman magnetic field results.

\begin{tabular}{lcccccc}
\hline \hline Cloud & $\begin{array}{c}B_{\text {los }} \\
\mathrm{mG}\end{array}$ & $\begin{array}{c}\sigma \\
\mathrm{mG}\end{array}$ & $\begin{array}{c}M / \Phi \\
\text { (wrt critical) }\end{array}$ & $\begin{array}{c}\mathcal{M} \\
10^{-9} \mathrm{erg} \mathrm{cm}^{-3}\end{array}$ & $\begin{array}{c}\mathcal{T} \\
10^{-9} \mathrm{erg} \mathrm{cm}^{-3}\end{array}$ & $\mathcal{M} / \mathcal{T}$ \\
\hline W3OH & 1.10 & 0.33 & $\leq 0.6$ & $\geq 48$ & 5.8 & $\geq 8$ \\
OMC1s & 0.04 & 0.24 & - & - & 16 & - \\
OMC1n1 & -0.36 & 0.08 & $\leq 4.5$ & $\geq 5.2$ & 7.8 & $\geq 0.7$ \\
OMC1n4 & 0.08 & 0.10 & - & - & 6.9 & - \\
NGC2024 & 0.01 & 0.12 & - & - & 6.3 & - \\
S255 & -0.73 & 0.34 & $\leq 2.2$ & $\geq 21$ & 4.3 & $\geq 5$ \\
G10.6 & 0.74 & 0.27 & $\leq 8.4$ & $\geq 22$ & 100 & $\geq 0.2$ \\
M17SWHI & 0.14 & 0.13 & - & - & 24 & - \\
M17SWCN & -0.22 & 0.08 & $\leq 14$ & $\geq 1.9$ & 14 & $\geq 0.1$ \\
S106OH & -0.52 & 0.38 & - & - & 0.9 & - \\
S106CN & -0.06 & 0.20 & - & - & 0.8 & - \\
DR21OH1 & -0.36 & 0.10 & $\leq 2.4$ & $\geq 5.2$ & 2.0 & $\geq 2.6$ \\
DR21OH2 & -0.71 & 0.12 & $\leq 1.0$ & $\geq 20$ & 2.0 & $\geq 10$ \\
S140 & -0.25 & 0.09 & $\leq 4.4$ & $\geq 2.5$ & 7.6 & $\geq 0.3$ \\
\hline
\end{tabular}

results near the $2 \sigma$ limit may not be true detections. There are therefore 8 probable detections of $B_{\text {los }}$ and 6 sensitive upper limits. Finally, we list the mass-to-magnetic flux ratios $M / \Phi$ with respect to critical and the magnetic and kinetic energy densities $\mathcal{M}=B^{2} / 8 \pi$ and $\mathcal{T}=\frac{1}{3} \rho \sigma^{2}$. Note that the quantities involving the magnetic field strength are determined from $B_{\mathrm{los}}$ and not $B_{\mathrm{tot}}$. $B_{\text {los }}$ is a lower limit to $B_{\text {tot }}$; hence, for detections of the magnetic field, $M / \Phi$ is an upper limit and $\mathcal{M}$ is a lower limit, as shown in the table. For non-detections of the Zeeman effect, we have a $2 \sigma$ upper limit on $B_{\text {los }}$; hence, we do not list values for the magnetic quantities in these cases.

\section{Comparison with other observations}

Toward S140 our result is $B_{\mathrm{los}}=-0.25 \pm 0.09 \mathrm{mG}$, a detection at the $2.8 \sigma$ level. Uchida et al. (2001) reported no detection in SO $J_{N}=1_{2}-1_{1}$ line Zeeman observation, with $\sigma\left(B_{\text {los }}\right) \approx 0.4 \mathrm{mG}$, which is consistent with our result. The critical density of the SO transition is about an order of magnitude higher than that of the $\mathrm{CN}$ transition, so SO samples higher density gas. The $\mathrm{CN}$ and SO observations were at the peak line strengths positions in each species, and differed by $\sim 30^{\prime \prime}$, or about half the SO beam width.

Toward $\mathrm{W} 3 \mathrm{OH}$ our result is $B_{\mathrm{los}}=+1.1 \pm 0.3 \mathrm{mG}$, a detection at the $3.7 \sigma$ level. Güsten et al. (1994) reported a Zeeman detection in their excited-state $\mathrm{OH}$ absorption line observations, and inferred $B_{\text {los }}=+3.2 \pm 0.6 \mathrm{mG}$ from a fit to the entire I profile. However, comparison of the results is complicated by the fact that the line profiles of $\mathrm{OH}$ and $\mathrm{CN}$ do not agree in detail. The $\mathrm{OH}$ line has two components, at -45.1 and $-47.5 \mathrm{~km} \mathrm{~s}^{-1}$. Güsten et al. felt that the Zeeman signal came from the stronger component at $-45.1 \mathrm{~km} \mathrm{~s}^{-1}$, and inferred $B_{\mathrm{los}}=+3.1 \pm 0.4 \mathrm{mG}$ from a fit to that component only. The $\mathrm{CN}$ line appears to have three velocity components, none of which agrees precisely with the $\mathrm{OH}$ components. These differences may be due to the fact that the $\mathrm{OH}$ absorption must come from our side of the continuum, while the $\mathrm{CN}$ emission may come from behind the continuum. Also, $\mathrm{CN}$ is seen in emission with a $23^{\prime \prime}$ beam, while the $\mathrm{OH}$ is in absorption against the $\sim 1^{\prime \prime}$ compact $\mathrm{H}$ II region. Moreover, the two transitions sample different densities. The $\Lambda$ doublet lines are within the ${ }^{2} \Pi_{3 / 2}, J=7 / 2$ state of $\mathrm{OH}$, which is $290 \mathrm{~K}$ above the ground state. The $\mathrm{OH}$ observations therefore sample hot, dense gas. Cesaroni \& Walmsley (1991) estimated $n\left(\mathrm{H}_{2}\right) \sim 7 \times 10^{6} \mathrm{~cm}^{-3}$ and $T_{\text {dust }} \approx 150 \mathrm{~K}$ for the region probed by the excited-state $\mathrm{OH}$ lines.
We observed two positions toward the M17SW molecular cloud. The M17SW(CN) position is at a local $\mathrm{CN}$ peak intensity position. That position was also observed during the earlier experiment (Crutcher et al. 1999), when $B_{\text {los }}=-0.33 \pm$ $0.14 \mathrm{mG}$ was reported. That is consistent with our new result of $B_{\text {los }}=-0.16 \pm 0.10 \mathrm{mG}$; combining the two observations yields $B_{\text {los }}=-0.22 \pm 0.08 \mathrm{mG}$. Our results can be compared with VLA H I and OH absorption-line Zeeman maps (Brogan \& Troland 2001) made with beams sizes close to our $\mathrm{CN}$ beam size. They did not report a detection in $\mathrm{H}$ I at our M17SW(CN) position, but an extrapolation from positions $\sim 1$ beam away suggests $B_{\text {los }} \approx-0.1 \mathrm{mG}$ in the $20 \mathrm{~km} \mathrm{~s}^{-1}$ velocity component, in fair agreement with our result. The M17SW(HI) position corresponds to a position where $B_{\text {los }} \approx-0.5 \mathrm{mG}$ in both $\mathrm{OH}$ and $\mathrm{H} \mathrm{I}$. The VLA result is completely different from our $\mathrm{CN}$ result of $B_{\text {los }}=+0.14 \pm 0.13 \mathrm{mG}$; although our $\mathrm{CN}$ result is not a detection, it differs by $\sim 5 \sigma$ from the VLA results. As for $\mathrm{W} 3 \mathrm{OH}$, the $\mathrm{CN}$ emission and $\mathrm{OH}$ and $\mathrm{HI}$ absorption lines may sample different regions.

\section{Discussion}

\subsection{Origin of the $\mathrm{CN}$ line emission}

Our targets are peaks of $\mathrm{CN} N=1 \rightarrow 0$ line emission in active star formation regions. In most cases, these peaks do not exactly coincide in projection with the center of dense cores, as traced by other molecular lines such as CS and isotopes or $\mathrm{HC}_{3} \mathrm{~N}$. This could perhaps be explained by the formation routes of CN. Boger \& Sternberg (2005) suggest that in gas denser than $10^{4} \mathrm{~cm}^{-3}$, the entire $\mathrm{CN}$ column density is built up in the $\mathrm{C}^{+} / \mathrm{C} / \mathrm{CO}$ transition layer and the larger the density, the sharper the concentration of the regions of large $\mathrm{CN}$ abundances in the illuminated layers $\left(A_{\mathrm{v}}<2 \mathrm{mag}\right)$. In this astrochemical picture, the $\mathrm{CN} N=1 \rightarrow 0$ transition with its critical density of the order of $10^{5} \mathrm{~cm}^{-3}$ is selectively sensitive to UV irradiated layers of dense cores, and/or dense PDRs. The CN Zeeman measurements would then sample a magnetic field strength that is not necessarily that present within the dense core itself. However, our inferrence of $\mathrm{CN}$ column densities seems to be at odds with this astrochemical picture.

Our analysis of the $\mathrm{CN}$ data yielded the $\mathrm{CN}$ column densities in the ground state, $N_{0}(\mathrm{CN})$; over the 14 positions the range was $2 \times 10^{13} \mathrm{~cm}^{-2}<N_{0}(\mathrm{CN})<9 \times 10^{13} \mathrm{~cm}^{-2}$, with a geometric mean $N_{0}(\mathrm{CN}) \approx 7 \times 10^{13} \mathrm{~cm}^{-2}$. Our LTE calculation of the column densities in all rotational states yielded 


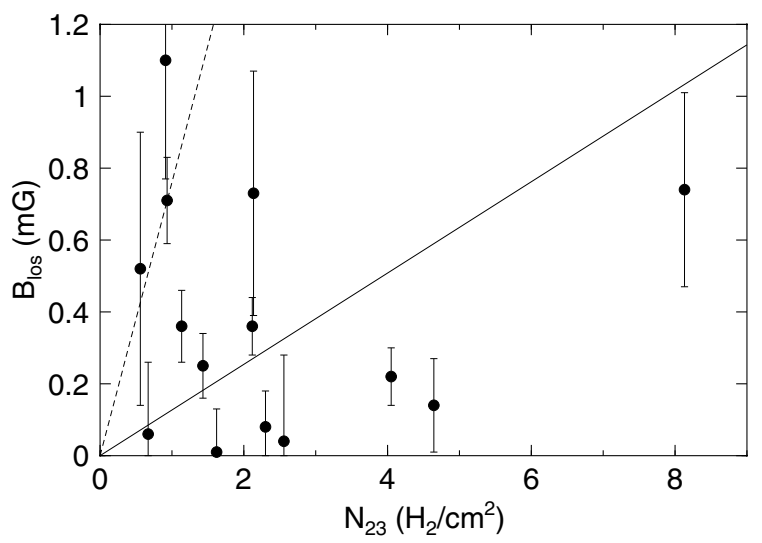

Fig. 2. The line-of-sight magnetic field strength from our CN Zeeman observations versus the column density of $\mathrm{H}_{2}$ (the geometric mean of our two determinations). $N_{23}$ is $N \times 10^{-23}$. The dashed line is the critical mass-to-flux ratio, and the solid line is the median mass-to-flux ratio inferred from $B_{\text {los }}$ (before geometric corrections).

$2 \times 10^{14} \mathrm{~cm}^{-2}<N(\mathrm{CN})<2 \times 10^{15} \mathrm{~cm}^{-2}$, with a geometric mean $N(\mathrm{CN}) \approx 6 \times 10^{14} \mathrm{~cm}^{-2}$. With $[\mathrm{CN}] /\left[\mathrm{H}_{2}\right]=4 \times 10^{-9}$, these values for $N(\mathrm{CN})$ yielded $N_{\mathrm{CN}}\left(\mathrm{H}_{2}\right)$ in reasonable agreement (i.e., within a factor 3) with $N_{n, r}\left(\mathrm{H}_{2}\right)$, derived from volume densities and radii. This agreement suggests that the value $[\mathrm{CN}] /\left[\mathrm{H}_{2}\right]$ we used is approximately correct. The values for $N(\mathrm{CN})$ and $[\mathrm{CN}] /\left[\mathrm{H}_{2}\right]$ do not agree with theoretical astrochemical values (cf. Boger \& Sternberg 2005), which are based on CN existing in PDR regions only. In particular, the model has significantly lower $N(\mathrm{CN})$ than the values we observe. Our results suggest that the $\mathrm{CN}$ in the regions we observe sample approximately the same regions and densities as sampled by CS, and therefore that $\mathrm{CN}$ serves as a good probe of magnetic field strengths in dense regions, $n\left(\mathrm{H}_{2}\right) \sim 10^{5-6} \mathrm{~cm}^{-3}$. This view may be supported by the recent findings of Hily-Blant et al. (2008) who show that $\mathrm{CN}$, like $\mathrm{N}_{2} \mathrm{H}^{+}$and unlike $\mathrm{CO}$, is not depleted towards the central parts of two dense cores.

\subsection{Mass to flux ratio}

Figure 2 shows our measured $B_{\text {los }}$ in $\mathrm{mG}$ versus $N_{23}\left(\mathrm{H}_{2}\right)$ in $10^{23} \mathrm{~cm}^{-2}$. We can use these values to compute the measured mass-to-flux ratios with respect to the critical value, $(M / \Phi)_{c r} \approx$ $0.76 N_{23}\left(\mathrm{H}_{2}\right) / B(\mathrm{mG})$ (cf. Crutcher 2004). The weighted (by uncertainties in $B_{\text {los }}$ ) mean value of the mass-to-flux ratio with respect to critical is $M / \Phi=6.0 \pm 0.5$. All measurements, including the non-detections, are included in this calculation. That mean value is plotted as a solid line. The dashed line shows the locus of critical mass-to-flux ratio; points above this line would have a subcritical $M / \Phi$. Keeping in mind that $B_{\text {los }}$ is a lower limit to $B_{\text {tot }}$, all observed points on this plot could be higher (less supercritical, more subcritical) if we could plot $B_{\text {tot }}$. Two points lie on the critical line and one lies above the critical line even with $B_{\text {los }}$ being plotted. This suggests that these cores are critical to subcritical, but the uncertainties in the measures of $B_{\text {los }}$ are too large for a definite result.

Although we can only determine upper limits on the mass-toflux ratios for individual sources, we can use our weighted mean value to carry out a statistical assessment. Heiles \& Troland (2005) have shown that the observed distribution of $B_{\text {los }}$ does constrain the median value of $B_{\text {tot }}$, and that the average value of $B_{\text {los }}$ is close to $B_{\text {tot }} / 2$ for a wide range of probability distributions of $B_{\text {tot }}$. We need to correct for the facts that we measure only the line-of-sight component of the magnetic field (the factor of $1 / 2$ correction discussed above), and that if the cores have a disk morphology we overestimate the column densities along the magnetic field (see Crutcher 2004). The combined correction for both of these geometry effects is $1 / 3$, so our best estimate for the mean mass-to-flux ratio in these cores is $M / \Phi \approx 2.0$ with respect to critical. Hence, our determination of the mean value of $M / \Phi$ yields a slightly supercritical result. However, the estimate of column densities is uncertain by $\sim 2$, so the possible range of the mean mass-to-flux ratio is $M / \Phi \sim 1-4$, or critical to supercritical by a factor of four.

\subsection{Alfvénic Mach number}

The Alfvénic Mach number is given by $M_{\mathrm{A}}=\sqrt{3} \sigma / v_{\mathrm{A}}$, where $\sigma$ is the one-dimensional velocity dispersion and $v_{\mathrm{A}}=B_{\text {tot }} / \sqrt{4 \pi \rho}$ is the Alfvén speed. Then $M_{\mathrm{A}}=5.1 \times 10^{-4} \sqrt{n\left(\mathrm{H}_{2}\right)} \Delta v / B_{\text {tot }}$, where $n\left(\mathrm{H}_{2}\right)$ is in $\mathrm{cm}^{-3}, \Delta v$ is in $\mathrm{km} \mathrm{s}^{-1}$, the total field strength $B_{\text {tot }}$ is in $\mathrm{mG}$, and we have assumed Gaussian velocity profiles and a $10 \% \mathrm{He}$ abundance. The geometric mean values from Tables 2 and 3 are $n\left(\mathrm{H}_{2}\right)=4.5 \times 10^{5} \mathrm{~cm}^{-3}, \Delta v=2.5 \mathrm{~km} \mathrm{~s}^{-1}$, and $B_{\text {los }}=0.28 \mathrm{mG}$. With the mean geometrical statistical correction $2 B_{\text {los }}=B_{\text {tot }}$, we have a mean Alfvénic Mach number $M_{\mathrm{A}}=1.5$. Therefore, turbulent and magnetic energies are in approximate equipartition.

\section{Conclusions}

These new observations of the Zeeman effect on the $\mathrm{CN} N=$ 1-0 lines have significantly improved the statistics on the magnetic field intensity in dense star formation regions. We use our 8 detections and 6 non-detections to derive a median value of the total field $B_{\mathrm{tot}}=0.56 \mathrm{mG}$ in gas of average density $n\left(\mathrm{H}_{2}\right)=4.5 \times 10^{5} \mathrm{~cm}^{-3}$. We show that the $\mathrm{CN}$ line probably samples regions similar to those traced by $\mathrm{CS}$ and are therefore confident that the magnetic field observed mostly pervades the dense cores. The dense cores are found to be critical to slightly supercritical with a mean mass-to-flux ratio $M / \Phi \sim 1$ to 4 , with respect to critical. Their turbulent and magnetic energies are in approximate equipartition, or the observed internal motions are slightly super-Alfvénic.

Since the Zeeman effect is sensitive only to the line-of-sight component of the field, more definite results can be obtained only if the statistical sample is larger. The range of densities of interest to star formation critically requires more $\mathrm{CN}$ Zeeman measurements (detections and sensitive upper limits).

Acknowledgements. This work was supported in part by the National Science Foundation under NSF grants AST 0205810, 0307642, and 0606822 and PHY 0551164. This paper is preprint number NSF-KITP-07-200. We are indebted to Pierre Hily-Blant for his help in preparing procedures with the New Control System at IRAM-30 m.

\section{Appendix A: Notes and references for individual sources}

W3OH: Distance from Reid et al. (2005), radius from Kim et al. (2006); close to H II region, 10" from submm source (Mueller et al. 2002). Density is inferred from the observations of $C^{34} S(3-2)$ and 5-4 by Plume et al. (1997).

OMC1s, OMC1n1, OMC1n4: Radii from Simon (1998), Tatematsu et al. (1993).

NGC2024: Radius from Simon (1998); density from CS multitransition analysis of Lada et al. (1997), adopting the 
mean logarithmic value of the density determined over the region emitting in $\mathrm{CS}(5-4)$. The $\mathrm{CN}$ position lies at the edge of the densest north-south ridge.

S255: Radius from Simon (1998); the CN position in S255 coincides with the dust continuum source SMM2, and the gas density is derived from this study (Minier et al. 2005).

G10.6: Radius from Ho et al. (1994); the $\mathrm{CN}$ position is that of the dust continuum emission peak (Mueller et al. 2002) and the density is that deduced from CS multi-transition analysis by Omodaka et al. (1992). This density is very close to that deduced from the density distribution found by Mueller et al. (2002) for that source, at half-power radius or $0.2 \mathrm{pc}$ from the center.

M17SWHI, M17SWCN: The CN peak in M17SW is at the edge of the dense PDR and the $\mathrm{H}_{2}$ density is inferred from the CS multitransition analysis of Wang et al. (1993). It is consistent with the density derived from $\mathrm{HC}_{3} \mathrm{~N}$ by Bergin et al. (1996). There is no indication, according to these tracers for a lower density at the position of M17SW(HI), 1.5' south of M17SW(CN).

S106CN, S106OH: Radius from Simon (1998); distance from Schneider et al. (2007); density from Vallée \& Fiege (2005).

DR21OH1, DR21OH2: Radius from Padin et al. (1989); density from Vallée \& Fiege (2006).

S140: The CN peak (Simon 1998) is $3^{\prime \prime}$ east, $5^{\prime \prime}$ south of SMM1 (Minchin et al. 1995); the density is inferred from the CS multitransition analysis of Zhou et al. (2004).

\section{References}

Bergin, E. A., Snell, R. L., \& Goldsmith, P. F. 1996, ApJ, 460, 343 Boger, G. I., \& Sternberg, A. 2005, ApJ, 632, 302

Brogan, C. L., \& Troland, T. H. 2001, ApJ, 560, 821

Cabrit, S. 2007, LNP, 723, 21

Cesaroni, R., \& Walmsley, C. M. 1991, A\&A, 241, 537
Crutcher, R. M. 2004, Ap\&SS, 292, 225

Crutcher, R. M. 2007, in Sky Polarisation at Far-Infrared to Radio Wavelengths: The Galactic Screen before the Cosmic Microwave Background, ed. M.-A. Miville-Deschênes, \& F. Boulanger, EAS Publication Series, 23, 37

Crutcher, R. M., Troland, T., Lazareff, B., \& Kazès, I. 1996, ApJ, 456, 217

Crutcher, R. M., Troland, T., Lazareff, B., Paubert, G., \& Kazès, I. 1999, ApJ, 514, L121

Güsten, R., Fiebig, D., \& Uchida, K. I. 1994, A\&A, 286, L51

Heiles, C., \& Troland, T. H. 2005, ApJ, 624, 773

Hily-Blant, P., Walmsley, M., Pineau des Forêts, G., \& Flower, D. 2008, A\&A, 480, L5

Ho, P. T. P., Terebey, S., \& Turner, J. L. 1994, ApJ, 423, 320

Johnstone, D., Boonman, A. M. S., \& van Dishoeck, E. F. 2003, A\&A, 412, 157

Kim, S.-J., Kim, H.-D., Lee, Y., et al. 2006, ApJS, 162, 161

Lada, E. A., Evans, N. J., II, \& Falgarone, E. 1997, ApJ, 488, 286

MacLow, M.-M., \& Klessen, R. S. 2004, Rev. Mod. Phys., 76, 125

Minchin, N. R., Ward-Thompson, D., \& White, G. J. 1995, A\&A, 298, 894

Minier, V., Burton, M. G., Hill, T., et al. 2005, A\&A, 429, 945

Mouschovias, T. Ch., \& Ciolek, G. E. 1999, in The Origin of Stars and Planetary Systems, ed. C. J. Lada, \& N. D. Kylafis (Kluwer), 305

Mueller, K. E., Shirley, Y. L., Evans, N. J., II, \& Jacobson, H. R. 2002, ApJS, 143,469

Omodaka, T., Kobayashi, H., Kitamura, Y., Nakano, M., \& Ishiguro, M. 1992, PASJ, 44, 447

Padin, S., Sargent, A. I., Mundy, L. G., et al. 1989, ApJ, 337, L45

Plume, R., Jaffe, D. T., Evans, N. J., II, Martin-Pintado, J., \& Gomez-Gonzalez, J. 1997, ApJ, 476, 730

Reid, M. J., Xu, Y., Zheng, X. W., \& Menten, K. M. 2005, A\&AS, 207, 4006

Schneider, N., Simon, R., Bontemps, S., Comerón, F., \& Motte, F. 2007, A\&A, 474,873

Shu, F., Allen, A., Shang, H., Ostriker, E. C., \& Li, Z.-Y. 1999, in The Origin of Stars and Planetary Systems, ed. C. J. Lada, \& N. D. Kylafis (Kluwer Academic Publishers), 193

Simon, R. 1998, private communication

Tatematsu, E., Umemoto, T., Kameya, O., et al. 1993, ApJ, 404, 643

Turner, B. E., \& Gammon, R. H. 1975, ApJ, 198, 71

Uchida, K.I., Fiebig, D., \& Güsten, R. 2001, A\&A, 371, 274

Vallée, J. P., \& Fiege, D. 2005, ApJ, 627, 263

Vallée, J. P., \& Fiege, D. 2006, ApJ, 636, 332

Wang, Y., Jaffe, D. T., Evans, N. J., II, et al. 1993, ApJ, 419, 707

Zhou, S., Butner, H. M., Evans, N. J., II, et al. 2004, ApJ, 428, 219 